\title{
Thermoreceptor TRPV1 regulates body weight and blood pressure in the absence of thermogenin
}

\author{
Shigeru Shibata ${ }^{1}$
}

Received: 10 January 2022 / Revised: 19 January 2022 / Accepted: 20 January 2022 / Published online: 2 March 2022

(c) The Author(s), under exclusive licence to The Japanese Society of Hypertension 2022

Although obesity is one of the frequent causes of high blood pressure, the molecular basis is not entirely clear owing to the heterogeneity of the two traits. Genetic, environmental, and behavioral factors all contribute to one being overweight, and increased salt sensitivity constitutes a key feature of obesity-related hypertension. In a subgroup of these patients, blood pressure and waist circumference correlate with plasma aldosterone, the overproduction of which could be attributable to humoral factors released from adipocytes $[1,2]$. There are also aldosterone-independent pathways that result in vascular dysfunction and renal salt retention, highlighting the complexity of the underlying mechanisms $[3,4]$.

Uncoupling protein 1 (UCP1), also known as thermogenin, regulates nonshivering thermogenesis, and modulates total body energy expenditure [5]. UCP1 is predominantly present in the inner mitochondrial membrane of brown adipose tissue (BAT) and allows the reentry of protons from the intermembrane space to the mitochondrial matrix. UCP1 diminishes the proton gradient formed by the electron transfer chain, bypassing ATP synthase and ultimately resulting in the generation of heat [5]. Physiologically, cold signals and sympathetic activation result in $\beta$-adrenergic stimulation of UCP1 in BAT, thereby triggering thermogenesis. This process is abrogated by the genetic ablation of Ucp 1, which is sufficient to increase body weight in mice [6]. Conversely, mice overexpressing Ucpl are resistant to obesity induced by a high-fat diet [7]. In addition, several clinical studies have reported the association of UCP1 with obesity and with hypertension in humans $[8,9]$, thus providing a possible mechanistic link between obesity and hypertension.

Shigeru Shibata

shigeru.shibata@med.teikyo-u.ac.jp

1 Division of Nephrology, Department of Internal Medicine, Teikyo University School of Medicine, Tokyo, Japan
Transient receptor potential vanilloid-1 (TRPV1) was identified in 1997 as a cation channel activated by capsaicin (the active substance in chili peppers) and thermal stimuli $\left(>43^{\circ} \mathrm{C}\right)[10]$, an achievement recognized by the Nobel Prize in Physiology or Medicine 2021. TRPV1 is now known to be activated by many other noxious stimuli, such as acid and toxins. In addition to nociception in sensory nervous systems, it regulates a number of physiological processes, including thermoregulation, sympathetic activity, and cardiovascular responses [11]. Sensory neurons expressing TRPV1 innervate adipose tissues, and TRPV1 is also present in adipocytes; indeed, clinical studies have indicated that TRPV1 can alter energy expenditure in BAT [12]. Nonetheless, the roles of TRPV1 in obesity and cardiovascular disorders remain controversial.

In this issue of Hypertens Res, Li et al. generated TRPV1 and UCP1 double knockout mice (Trpv1 $\left.1^{-/-} / \mathrm{Ucp}^{-/-}\right)$and analyzed the phenotype in detail (Fig. 1) [13]. In wild-type mice, neither body weight nor blood pressure were altered by the deletion of Trpvl. In $U c p 1^{-/-}$mice, however, the authors demonstrated that the knockout of Trpvl aggravated

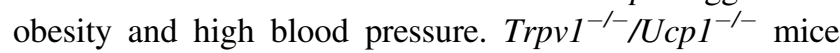
showed reduced oxygen consumption and heat production, which was accompanied by increased lipid content in the adipose tissues and an increase in abdominal circumference. In the BAT of $\operatorname{Trpv1}^{-/} / \mathrm{Ucpl}^{-/-}$mice, impaired mitochondrial function was suggested by the reduction in key proteins involved in BAT differentiation and lipolysis, altered oxidative phosphorylation, and impaired $\mathrm{Ca}^{2+}$ signaling in isolated BAT mitochondria. The authors further demonstrated that leucine-zipper-EF-hand containing transmembrane protein 1 (LETM1), a $\mathrm{Ca}^{2+} / \mathrm{H}^{+}$antiporter that is critical for mitochondrial homeostasis [14], is involved in these processes; they found that LETM1 and UCP1 levels were higher in $\operatorname{Trpv1^{-/}}$ mice than in wild-

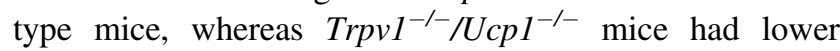
levels of LETM1 than $U c p 1^{-/-}$mice. The knockdown experiments indicated that the upregulation of LETM1 plays a compensatory role in the BAT of Trpv1 ${ }^{-/-}$mice, 


\section{Graphical Opinion}
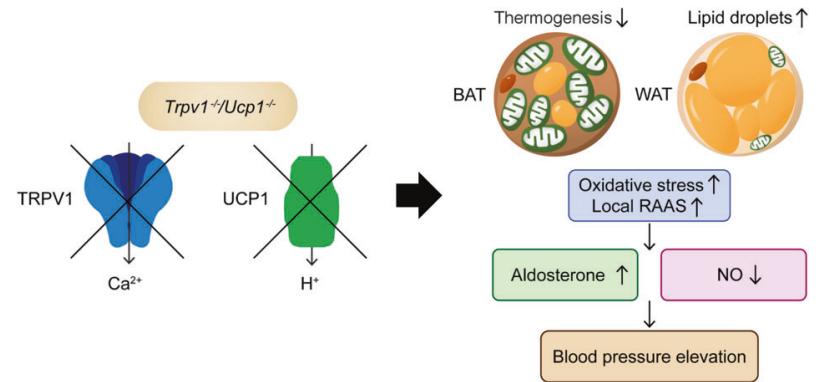

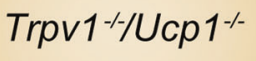

TRPV1

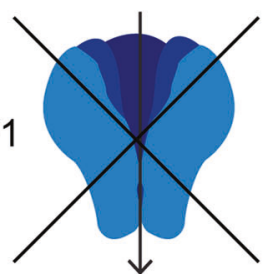

$\mathrm{Ca}^{2+}$
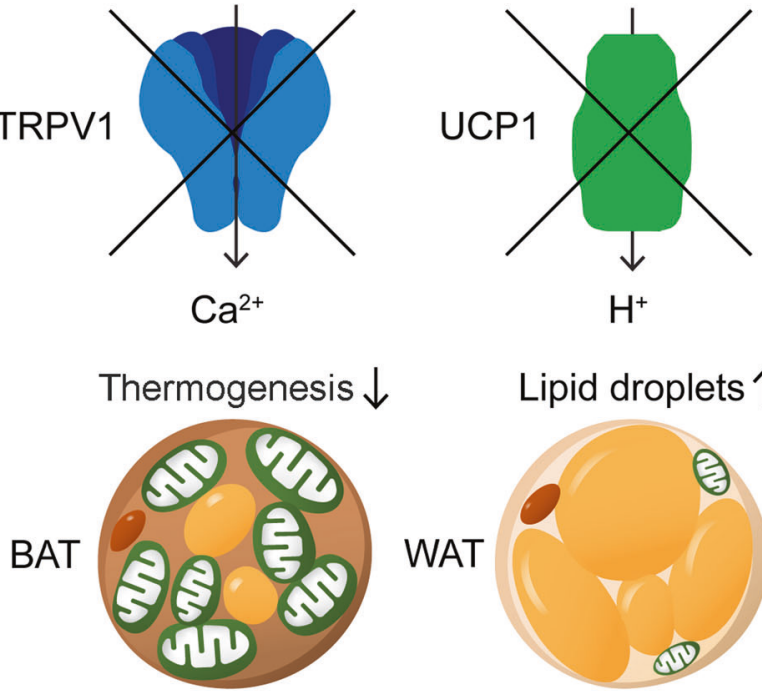

$\mathrm{H}^{+}$

WAT
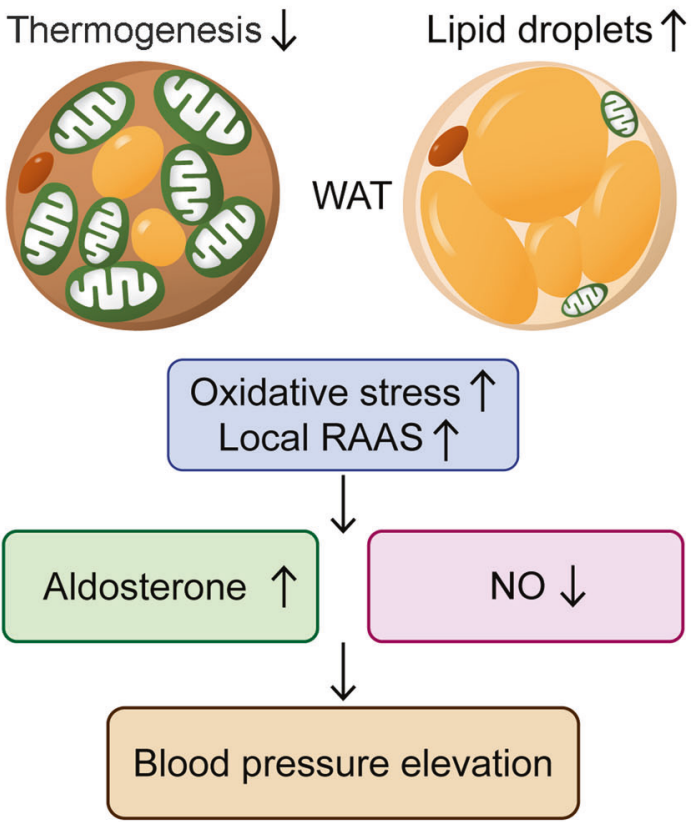

Fig. 1 Adipose tissue dysfunction in $\operatorname{Trp} v 1^{-/-} / U c p 1^{-/-}$mice results in hypertension through increased aldosterone and decreased nitric oxide (NO). TRPV1 transient receptor potential vanilloid-1, UCP1 uncoupling protein 1, BAT brown adipose tissue, WAT white adipose tissue, RAAS renin-angiotensin aldosterone system

and the process was impaired in the double knockout mice.

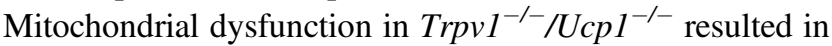
an increase in oxidative stress and mineralocorticoid receptor (MR) locally in the BAT, and it caused an increase in aldosterone and a decrease in NO bioavailability in the systemic circulation (Fig. 1). These changes likely explain the elevated blood pressure levels in $\operatorname{Trpv1} 1^{-/-} / \mathrm{Ucpl}^{-/-}$ mice.

The study by $\mathrm{Li}$ et al. provides additional clues to the pathogenesis of obesity-related hypertension and raises several provocative questions [13]. From the current study, it is unclear whether the blood pressure effects observed in

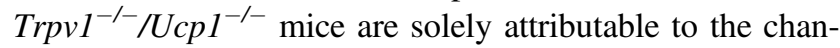
ges in adipose tissues or to signaling in other tissues, such as the vasculature, adrenal gland, nerve systems, and kidney; indeed, previous studies have shown that the modulation of TRPV1 signaling alters sympathetic nerve activity, vasoconstriction, and urinary sodium excretion [15-17]. It will be of interest to pursue the individual contributions in future studies. Additionally, the study showed that MR abundance in adipose tissues, as well as systemic aldosterone, was significantly increased in Trpv $1^{-/-} / U_{c p 1^{-/-}}$mice. In relation to this finding, recent studies have shown that finerenone, a nonsteroidal MR antagonist, improved metabolic parameters in high-fat dietfed mice, which was mediated by the induction of UCP1 and peroxisome proliferator-activated preceptor gamma coactivator 1 alpha (PGC-1 $\alpha)$ in the BAT [18]. Of note, it has been suggested that steroidal MR antagonists and nonsteroidal MR antagonists can differ in their tissue distribution patterns and in the binding modes to the receptor [19], which may result in differences in clinical effects. In support of this possibility, experimental studies have shown that the response of thermogenic markers in BAT was significantly different between finerenone and spironolactone in mice receiving a high-fat diet [20]. Given these data, how available MR antagonists alter the pathologies observed in

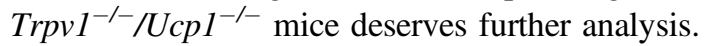

In patients with diabetes mellitus and hypertension, sodium glucose cotransporter 2 (SGLT2) inhibitors, such as empagliflozin, have been shown to produce clinically significant blood pressure reduction, especially in those with salt-sensitive hypertension [21, 22]. In addition, experimental studies have shown that SGLT2 inhibitors can reduce the salt sensitivity of blood pressure [23]. Given the 
previous data suggesting that SGLT2 inhibitors promote browning and lipolysis in adipose tissues [24], it is also of interest to address whether SGLT2 inhibition can attenuate adipocyte changes and local renin-angiotensin aldosterone system (RAAS) activation in this model.

In summary, the study by $\mathrm{Li}$ et al. showed that the deletion of the cation channel TRPV1 in mice lacking UCP1 compromises mitochondrial function in BAT, resulting in body weight gain. This effect is accompanied by local RAAS activation, aldosterone excess, and hypertension. These data provide novel information regarding the link among obesity, aldosterone, and high blood pressure, which can be further characterized in future studies.

Acknowledgements We thank Editage for creating the artwork.

Funding This work was supported in part by grants from the Ministry of Education, Culture, Sports, Science and Technology of Japan 19H03678. This work was also supported by Advanced Comprehensive Research Organization (ACRO) Research Grants from Teikyo University.

\section{Compliance with ethical standards}

Conflict of interest SS has received honoraria from Daiichi Sankyo outside the submitted work.

Publisher's note Springer Nature remains neutral with regard to jurisdictional claims in published maps and institutional affiliations.

\section{References}

1. Kidambi S, Kotchen JM, Grim CE, Raff H, Mao J, Singh RJ, et al. Association of adrenal steroids with hypertension and the metabolic syndrome in blacks. Hypertension. 2007;49:704-11.

2. Nagase M, Yoshida S, Shibata S, Nagase T, Gotoda T, Ando K, et al. Enhanced aldosterone signaling in the early nephropathy of rats with metabolic syndrome: possible contribution of fat-derived factors. J Am Soc Nephrol. 2006;17:3438-46.

3. DeMarco VG, Aroor AR, Sowers JR. The pathophysiology of hypertension in patients with obesity. Nat Rev Endocrinol. 2014;10:364-76.

4. Ishizawa K, Wang Q, Li J, Xu N, Nemoto Y, Morimoto C, et al. Inhibition of sodium glucose cotransporter 2 attenuates the dysregulation of Kelch-like 3 and $\mathrm{NaCl}$ cotransporter in obese diabetic mice. J Am Soc Nephrol. 2019;30:782-94.

5. Lowell BB, Spiegelman BM. Towards a molecular understanding of adaptive thermogenesis. Nature. 2000;404:652-60.

6. Feldmann HM, Golozoubova V, Cannon B, Nedergaard J. UCP1 ablation induces obesity and abolishes diet-induced thermogenesis in mice exempt from thermal stress by living at thermoneutrality. Cell Metab. 2009;9:203-9.

7. Li B, Nolte LA, Ju JS, Han DH, Coleman T, Holloszy JO, et al. Skeletal muscle respiratory uncoupling prevents diet-induced obesity and insulin resistance in mice. Nat Med. 2000;6:1115-20.

8. Nagai N, Sakane N, Ueno LM, Hamada T, Moritani T. The -3826 A $\rightarrow \mathrm{G}$ variant of the uncoupling protein-1 gene diminishes postprandial thermogenesis after a high fat meal in healthy boys. $\mathrm{J}$ Clin Endocrinol Metab. 2003;88:5661-7.
9. Sun H, Zhang JT, Xie XR, Li T, Li XY, Wang NN, et al. Association of uncoupling protein gene polymorphisms with essential hypertension in a northeastern Han Chinese population. J Hum Hypertens. 2019;33:524-30.

10. Caterina MJ, Rosen TA, Tominaga M, Brake AJ, Julius D. A capsaicin-receptor homologue with a high threshold for noxious heat. Nature. 1999;398:436-41.

11. Gladkikh IN, Sintsova OV, Leychenko EV, Kozlov SA. TRPV1 ion channel: structural features, activity modulators, and therapeutic potential. Biochemistry. 2021;86:S50-70.

12. Yoneshiro T, Aita S, Kawai Y, Iwanaga T, Saito M. Nonpungent capsaicin analogs (capsinoids) increase energy expenditure through the activation of brown adipose tissue in humans. Am J Clin Nutr. 2012;95:845-50.

13. Li L, Ma L, Luo Z, Wei X, Zhao Y, Zhou C, et al. Lack of TRPV1 aggravates obesity-associated hypertension through the disturbance of mitochondrial $\mathrm{Ca} 2+$ homeostasis in brown adipose tissue. Hypertens Res. 2022. https://doi.org/10.1038/s41440-021-00842-8.

14. Jiang D, Zhao L, Clapham DE. Genome-wide RNAi screen identifies Letm1 as a mitochondrial $\mathrm{Ca} 2+/ \mathrm{H}+$ antiporter. Science. 2009;326:144-7.

15. Yu SQ, Ma S, Wang DH. Selective ablation of TRPV1 by intrathecal injection of resiniferatoxin in rats increases renal sympathoexcitatory responses and salt sensitivity. Hypertens Res. 2018;41:679-90.

16. Phan TX, Ton HT, Gulyas H, Porszasz R, Toth A, Russo R, et al. TRPV1 expressed throughout the arterial circulation regulates vasoconstriction and blood pressure. J Physiol. 2020;598:5639-59.

17. Li L, Wang F, Wei X, Liang Y, Cui Y, Gao F, et al. Transient receptor potential vanilloid 1 activation by dietary capsaicin promotes urinary sodium excretion by inhibiting epithelial sodium channel alpha subunit-mediated sodium reabsorption. Hypertension. 2014;64:397-404.

18. Marzolla V, Feraco A, Gorini S, Mammi C, Marrese C, Mularoni $\mathrm{V}$, et al. The novel non-steroidal MR antagonist finerenone improves metabolic parameters in high-fat diet-fed mice and activates brown adipose tissue via AMPK-ATGL pathway. FASEB J. 2020;34:12450-65.

19. Kolkhof P, Delbeck M, Kretschmer A, Steinke W, Hartmann E, Barfacker L, et al. Finerenone, a novel selective nonsteroidal mineralocorticoid receptor antagonist protects from rat cardiorenal injury. J Cardiovasc Pharmacol. 2014;64:69-78.

20. Marzolla V, Feraco A, Limana F, Kolkhof P, Armani A, Caprio M. Class-specific responses of brown adipose tissue to steroidal and nonsteroidal mineralocorticoid receptor antagonists. J Endocrinol Investig. 2022;45:215-20.

21. Kario K, Okada K, Kato M, Nishizawa M, Yoshida T, Asano T, et al. 24-Hour blood pressure-lowering effect of an SGLT-2 inhibitor in patients with diabetes and uncontrolled nocturnal hypertension: results from the randomized, placebo-controlled SACRA study. Circulation. 2018;139:2089-97.

22. Ferdinand KC, Izzo JL, Lee J, Meng L, George J, Salsali A, et al. Antihyperglycemic and blood pressure effects of empagliflozin in black patients with Type 2 diabetes mellitus and hypertension. Circulation. 2019;139:2098-109.

23. Wan N, Fujisawa Y, Kobara H, Masaki T, Nakano D, Rahman A, et al. Effects of an SGLT2 inhibitor on the salt sensitivity of blood pressure and sympathetic nerve activity in a nondiabetic rat model of chronic kidney disease. Hypertens Res. 2020;43:492-9.

24. Xu L, Nagata N, Nagashimada M, Zhuge F, Ni Y, Chen G, et al. SGLT2 inhibition by empagliflozin promotes fat utilization and browning and attenuates inflammation and insulin resistance by polarizing M2 macrophages in diet-induced obese mice. EBioMedicine. 2017;20:137-49. 